\title{
Flores, jardines y bosques: la búsqueda de autoridades en $P$. Mexía de Torquemada, L. Zapata y J. Pérez de Moya
}

Strosetzki, Christoph

First published in:

Loca ficta, S. 413 - 426, Vervuert, Madrid 2003, ISBN 3-89354-418-6

Münstersches Informations- und Archivsystem multimedialer Inhalte (MIAMI) URN: urn:nbn:de:hbz:6-21479465272 


\title{
FLORES, JARDINES Y BOSQUES: \\ LA BÚSQUEDA DE AUTORIDADES EN P. MEXÍA, A. DE TORQUEMADA, L. ZAPATA Y J. PÉREZ DE MOYA
}

\author{
Christoph Strosetzki \\ Universitat Münster
}

En el Renacimiento gozaron de especial popularidad las colecciones y los tomos de consulta como los "specula», los "florilegia», "compendia» o "polyanthea", que por una parte transmitían la erudición de la Antigüedad, y por otra, tenían que satisfacer los intereses de la nueva época'. El humanista «dotó de una paradójica 'novedad' a las autoridades antiguas ${ }^{2}$. Dentro de estas colecciones hay una diferencia entre los cuadernos loci communes $^{3}$, que a parte de ellos les basta con la recopilación de citas, y las colecciones de fenómenos extraordinarios del pasado y del presente. Al último grupo pertenecen la Silva (1540) de P. Mexía así como el Jardín de Flores Curiosas (1570) de A. de Torquemada ${ }^{5}$, los Miscelánea (1859) de L. Zapata ${ }^{6}$ y la Silva (1579) de J. Pérez de Moya?. Estos textos serán, a pesar de sus diferencias, tratados a continuación como

${ }^{1}$ Ver Infantes, 1988, p. 246.

${ }^{2}$ Ver Lerner, 1993 , p. 109.

${ }^{3}$ Relaciones entre «silva», especialmente «florilegium» con «loci» se encuentran en títulos como: de Granada, Fray L., Silva locorum, Salamanca, 1586; Lange, J., Loci communes sive Florilegium rerum, et materiarum ex auctoribus vetustis; theologis, philosophie, oratoribus, historicis, poetis etc., Straßburg, 1624, citado según López Poza, A., 1990 , pp. 69-70.

${ }^{4}$ Mexía, Silva de varia lección, t. 1 y 2.

${ }^{5}$ Torquemada, Jardin de flores Curiosas.

${ }^{6}$ Zapata, Miscelánea. Tomo XI del Memorial Histórico Español.

${ }^{7}$ Pérez de Moya, Silva Eutrapelias. Traducción de la primera edición de Valladolid 1557. 
un único Corpus. El género de la Memorabilia, en cuya tradición se incluyen ${ }^{8}$, debe entretener e informar, es decir, al mismo tiempo cumplir con los postulados de la historiografia y los de la literatura de ficción. La literatura de los Miscelánea como continuación de los Memorabilia, ofrece agradable entretenimiento, sin que por ello sea necesario inventar mundos imaginarios o figuras que puedan oponerse a la realidad histórica $^{9}$. Los autores de los Miscelánea insisten repetidas veces en que su pretensión no es inventar, sino presentar todo lo visto y todo lo oído, o por lo menos lo transmitido de una fuente segura ${ }^{10}$.

Los diferentes fenómenos dignos de mención se presentan en contextos diferentes: si con Mexía uno tiene la impresión de encontrarse en una biblioteca, con Torquemada, en cambio, uno se imagina una íntima conversación filosófica en un agradable jardín, mientras que con Zapata uno se halla en presencia de, según Rodríguez Cacho, "charlistas de café", los cuales tienen como finalidad entretener a un grupo de amigos con impresiones de viajes, libros y vivencias personales ${ }^{11}$. En Pérez de Moya las nociones son sobre todo prácticas y de utilidad, y además despiertan el interés por ser modernas ${ }^{12}$. Los Miscelánea ofrecían al lector al mismo tiempo compilaciones enciclopédicas de saber práctico para la conversación o el discurso. Eran, incluso, comprensibles por aquellos que no podían leer libros en latín, lenguaje científico, y echaban mano primeramente de las enciclopedias del siglo xviI o las revistas posteriores del tipo Reader's Digest. Los éxitos editoriales eran tales porque posibilitaban conocer en los miscelánea, para qué era preciso normalmente la lectura de muchos libros ${ }^{13}$. J. L. Vives, en cambio, ve en esta práctica de la desatención de fuentes originales una causa por la cual se produce el, por él tantas veces lamentado, retroceso de las ciencias ${ }^{14}$. Como humanista prefiere buscar la verdad en las fuentes que, a su juicio, son más seguras.

${ }^{8}$ La expresión «memorabilia» se encuentra también en el título de un libro como De dictis factisque memorabilibus collectanea, que Battista Fregoso, natural de Genua, publicó en 1508. Ver Infantes, 1988, p. 252; ver también respecto a la «Memorabile» Jolles, 1958, pp. 200-217.

${ }^{9}$ Ver Castro, 1989-1990, pp. 62-63.

${ }^{10}$ Ver Rallo Gruss, A., 1984, p. 167.

${ }^{11}$ Ver Rodríguez Cacho, 1993, p. 166.

12 Infantes, 1996, XV y XX.

${ }^{13}$ Ver Rodríguez Cacho, 1993, p. 156.

${ }^{14}$ Ver López Poza, 1990, p. 64. 
Los fenómenos y sucesos que se presentan en las colecciones citadas corresponden al postulado retórico y poetológico de la admiratio ${ }^{15}$. Precisamente lo inusual y lo extraordinario despiertan la curiosidad del lector ${ }^{16}$ y puesto que contradice la forma de pensar cotidiana, se convierte, por una parte, en algo más interesante; por la otra, se convierte a la vez en algo más improbable e increíble. Debido a que los fenómenos presentados despiertan el interés tan sólo si no son mera ficción literaria, sino que forman parte de la realidad, se tiene que desarrollar una estructura de argumentación para probar la veracidad de lo que se cuenta. Los criterios que se consideran válidos son especialmente instructivos para lo que se entiende en la España de comienzos de la edad moderna como verdad.

No es lo conocido, sino lo desconocido lo que causa admiración. Son las cosas «dignas de admiración, por no haber sido otras veces vistas ni oídas ${ }^{17}$. También lo insólito provoca asombro. Y así elige Mexía «materias que no fuessen muy comunes ni anduviessen por el vulgo» ${ }^{18}$. Se busca lo insólito porque lo usual no le interesa a nadie: "[S]i hombre no cuenta cosas grandes, las comunes nadie las quiere oír, aunque no las haya oído» ${ }^{19}$. Lo que parece inusual y fantástico, no es desde el principio imposible, como acentúa Torquemada ${ }^{20}$. También para Zapata debe ser extraordinario, pero sólo si también es verdad ${ }^{21}$. Que dado el tamaño del mundo haya todavía una cantidad infinita de materias y circunstancias desconocidas hasta el momento, ensombrece, según Torquemada, lo que ya se tiene por extraordinario ${ }^{22}$. Debido a que al sabio se le caracteriza por su interés por lo nuevo, aparece como el lector idea ${ }^{23}$. Siempre es precisamente lo inusual, lo no cotidiano, lo que

${ }^{15}$ En cuanto al significado de "admiratio» del lector frente a las «maravillas» ver Rodríguez Cacho, 1993, p. 159; ver también Lausberg, 1973, p. 58 y p. 180.

${ }^{16}$ Materias de la Miscelánea son: "Casos extraordinarios, narraciones ejemplares, agudezas, se combinan con acontecimientos científicos que van de experiencias fisicas a teorías sobre el comportamiento natural de animales y hombres extraños» (Rallo Gruss, 1987, p. 128).

17 Torquemada, Jardín de Flores Curiosas, p. 94 y p. 120.

${ }^{18}$ Mexía, Silva de varia lección, t. 1, p. 164 y p. 685.

${ }^{19}$ Mexía Silva de varia lección, t. 1, p. 679.

${ }^{20}$ Torquemada, Jardín de Flores Curiosas, p. 187.

${ }^{21}$ Zapata, Miscelánea, Tomo XI del Memorial Histórico Español, pp. 3-4 y p. 245.

22 Torquemada, Jardín de Flores Curiosas, p. 149 y p. 121.

${ }^{23}$ Torquemada, Jardín de Flores Curiosas, pp. 192-193. 
se debe elegir para satisfacer la intención poetológica de despertar la curiosidad en el lector. Pero siempre la condición es que lo insólito se pueda, en principio, catalogar como posible y verdadero.

La oposición entre la verdad y el interés en lo extraordinario hace pensar en los diferentes principios de los géneros de la historiografia y la literatura de ficción. Al lado «de cualquier cosa mínima [...] del curso ordinario» ${ }^{24}$ de la historiografia, argumenta Zapata: «Pues de cosas maravillosas el lector gusta» ${ }^{25}$. Precisa su intención: "[...] acarrear al lector cosas que le den gusto, aunque sean agenas» ${ }^{26}$. Si domina el principio del "delectare», que falta en la historiografia, entonces se sobrepasan fácilmente los límites con la literatura, como Zapata ve claramente $^{27}$. Mexía explica utilizando el ejemplo de un duelo entre dos caballeros en la época de Alfonso X, que lo verdadero puede entretener más que lo ficticio: «[L]os cuentos [...] que yo agora contaré, que son verdaderos y no menos estraños que los fingidos» ${ }^{28}$. En efecto, uno está acostumbrado a encontrar caballeros excepcionales y sus hazañas en los libros de caballerías. Por otra parte, Zapata acentúa que en el género dominado por lo extraordinario, los Miscelánea, no todo lo ficcional tiene que ser imposible:

Aunque los libros de caballerías mienten, pero los buenos autores vanse á la sombra de la verdad, aunque de la verdad á la sombra vaya mucho. Dicen que hendieron el yelmo, ya se ha visto; y que cortaron las mallas de las lorigas, ya tambien en nuestros tiempos se ha visto ${ }^{29}$.

Si aún se habla de mentira en sentido figurado en relación con los libros de caballerías, entonces el reproche de mentira está al alcance de la mano si se cuenta algo extraordinario. Tienen que ser preservadas de este reproche las cosas "que parecen mentira y son verdad" ${ }^{30}$.

${ }^{24}$ Zapata, Miscelánea, Tomo XI del Memorial Histórico Español, p. 191.

${ }^{25}$ Zapata, Miscelánea, Tomo XI del Memorial Histórico Español, p. 85.

${ }^{26}$ Zapata, Miscelánea, Tomo XI del Memorial Histórico Español, p. 177.

27 "Aunque parezca este cuento de Isopo, todavía lo diré.» (Zapata, Miscelánea, Tomo XI del Memorial Histórico Español, p. 328); ver también "[...] esto no es fábula, sino muy averiguado" (Torquemada, Jardín de Flores Curiosas, p. 112).

${ }^{28}$ Mexía, Silva de varia lección, t. 2, p. 177.

${ }^{29}$ Zapata, Miscelánea, Tomo XI del Memorial Histórico Español, p. 20; en cuanto al acercamiento a los libros de caballerías en Torquemada, ver Prieto, 1986, p. 255.

${ }^{30}$ Zapata, Miscelánea, Tomo XI del Memorial Histórico Español, p. 63. 
Por el contrario, los charlatanes están acostumbrados a entretener «el ánimo de los cortesanos creando abierta admiración» a costa de la verdad $^{31}$. Pérez de Moya se distancia de las historias de mentiras en interés de la verdad ${ }^{32}$. Cuando Mexía, de la infinita cantidad de cosas "más apartadas que otras de las comunes", quiere presentar las que le parecen "más estrañas y singulares», se distancia por un igual de ficción y mentira: «[...] y no contaré fábulas ni mentiras, sino lo que en auctores aprovados he leído" ${ }^{33}$.

¿Cómo se puede probar que en un fenómeno extraordinario concreto no se trata de un hecho ficticio o de una mentira, sino de la verdad? ¿Cuándo se puede juzgar por un caso aislado la verdad general? Torquemada aconseja precaución e introduce el refrán español de que una golondrina no hace verano, para ilustrar "que ésas son cosas que acaecen raramente, y no contradicen a una generalidad tan grande» ${ }^{34}$. Por ello, un sólo ejemplo no es suficiente para confirmar la verdad. Esto tiene validez, según Torquemada, sobre todo para los fenómenos extraordinarios: «Maravillas son esas que requieren que aleguéis en prueba de ellas tantos testigos como decís $\rangle^{35}$.

Se puede hablar de experiencia - un término que utiliza Torquemada $^{36}$ y que Pérez de Moya acentúa especialmente-, si se confirma varias veces la verdad de un hecho aislado: "He incluido en este libro muchas cosas que he experimentado yo mismo con mis instrumentos ${ }^{37}$. Pedro Mexía se ve, en cambio, en la necesidad de completar la experiencia personal con la ajena y resalta la importancia de un número lo más elevado posible de testigos para poder confirmar la verdad ${ }^{38}$, que pueden ser, sin embargo, tomados de los libros. Por tanto, M. D. Johnston no tiene razón, cuando contrapone en su comentario «el escrutinio de los textos a la observación de las co-

31 Zapata, Miscelánea, Tomo XI del Memorial Histórico Español, p. 63.

${ }^{32}$ Ver Pérez de Moya, Silva Eutrapelias. Traducción de la primera edicción de Valladolid 1557 , p. 3.

${ }_{33}$ Mexía, Silva de varia lección, t. 1, p. 407.

${ }^{3+}$ Torquemada, Jardín de Flores Curiosas, p. 111.

35 Torquemada, Jardín de Flores Curiosas, p. 133.

${ }^{36}$ "[...] tienen por cosa muy averiguada y conocida por experiencia, que [...]" (Torquemada, Jardín de Flores Curiosas, p. 114).

${ }^{37}$ Pérez de Moya, Silva Eutrapelias. Traducción de la primera edicción de Valladolid 1557 , p. 3 y p. 7.

${ }^{38}$ Ver Mexía, Silva de varia lección, t. 1, p. 607 y p. 734. 
sas», o bien "el estudio como ocio a la investigación como trabajo»" ya que el resultado de una observación puede ser transmitida tanto oralmente como por escrito.

En efecto, el testimonio de testigos frecuentemente aparece por escrito en forma de libro, así se completa la propia experiencia por medio de la lectura de otros autores, como en Pérez de Moya, el cual, como anuncia en el título de su obra, quiere partir de igual modo de «diversos autores de autorizado crédito y también de experiencias adquiridas». Si se exponen diferentes opiniones ajenas, para Torquemada es la propia experiencia, la que es decisiva en última instancia ${ }^{40}$.

Aunque la experiencia personal parece siempre como decisiva y por ello tiene supremacía sobre lo leído, se ve al mismo tiempo que no pocas veces se le interponen barreras espaciales y temporales que hacen indispensable limitarse a lo leído: con respecto a la existencia de los pigmeos, cita Torquemada a Antonio Guberto, el cual escribió que la existencia de los pigmeos fue inventada. Guberto justifica su opinión en la carencia de experiencia personal: "[C]on nunca se haber caminado tanta parte de él, en ninguna parte se hallen ni se sepa ahora que hay pigmeos $»^{41}$. Esta falta de experiencia no puede, sin embargo, convencer a Torquemada, ya que hay testimonios escritos de más importancia: «Pero dejando aparte a todos los otros que escribieron de esta materia, temeridad sería querer contradecir a tan graves autores como son Aristóteles y Solino y Plinio» ${ }^{42}$. Así sé demuestra que, por lo menos, la falta de experiencia puede tener menos fuerza probatoria que un testimonio escrito. Depende, por tanto, de los determinados casos, si la experiencia propia o la ajena es de mayor relevancia. Precisamente cuando se trata de «fantasmas y visiones», se enfrentan, según Zapata, dos posiciones: «unos que no creen que las hay, y dicen toda mi vida he andado de noche, y no he topado ninguno; y otros que creen que las hay, como es justo, de que están los libros llenos de ejemplos» ${ }^{43}$.

${ }^{39}$ Ver Johnston, 1978, pp. 72-73.

40 "[...] una cosa tan extraña, y que tiene necesidad de ser vista y averiguada por los ojos para acabar de ser bien entendida la verdad de ella» (Torquemada, Jardin de Flores Curiosas, pp. 408-409).

${ }^{41}$ Torquemada, Jardín de Flores Curiosas, p. 139.

42 Torquemada, Jardín de Flores Curiosas, p. 139.

${ }^{43}$ Zapata, Miscelánea, Tomo XI del Memorial Histórico Español, p. 442; para entender el significado de la experiencia personal en Zapata, ver Prieto, 1986, p. 239. 
Las experiencias e informaciones ajenas no se toman tan sólo de los libros sino también de lo que se oye de «muchas personas dignas de fe ${ }^{44}$. De todas formas, en tal indicación tan difusa de la fuente existe el peligro de incurrir en la falta de compromiso. Eso igualmente sirve para Mexía cuando escribe: "Notoria cosa es que»" ${ }^{45}$ "Y esta razón es muy poderosa y no se puede negar, pues se vee y se sabe» ${ }^{46}$. Torquemada no se compromete tampoco aunque en la forma parezca que sí lo haga. Él tiene por verdaderos los informes de los demás "pues que en nuestros días se dijo y afirmó por cosa muy averiguada $y$ verdadera ${ }^{47}$, o "si es verdad, como ya lo voy creyendo, pues fue negocio tan público y con tantos testimonios» ${ }^{48}$. Sin embargo, aquí se muestra que algo en la medida en que se documenta universalmente, pierde al mismo tiempo el carácter de extraordinario. A esto se añade que las habladurías de la gente no pueden demostrar un hecho, por eso, Torquemada lo desestima por parecerle falso, "que se trata en el vulgo, que, por la mayor parte son cosas fabulosas ${ }^{49}$. No todo informante es, por tanto, merecedor de crédito por igual, ya bien sean testigos o autores. En un pasaje Zapata previene incluso a los lectores de creer lo que a él, por otra parte, informantes fidedignos le han presentado como cierto $^{50}$ y en otro pasaje advierte contra el prejuicio, según el cual cuanto más alta sea la posición social de un informante, lo sea también su credibilidad ${ }^{51}$. Mexía desconfía de los relatos «de personas livianas y de poca auctoridad, de las quales muchas cosas he oído contar en este propósito» y prefiere, en cambio, «lo que dizen o escriven personas graves y de gran cuenta, y entre ellos, Plinio" ${ }^{52}$. Sin embargo, una opinión que es defendida al mismo tiempo por el pueblo y por muchos autores, siempre la considera creíble ${ }^{53}$. Según Torquemada, ya es suficiente una gran cantidad de autores como prue-

${ }^{44}$ Torquemada, Jardín de Flores Curiosas, p. 152.

${ }^{45}$ Mexía, Silva de varia lección, t. 1, p. 411.

${ }^{46}$ Mexía, Silva de varia lección, t. 1, pp. 514-515.

${ }^{47}$ Torquemada, Jardín de Flores Curiosas, p. 124.

${ }^{48}$ Torquemada, Jardín de Flores Curiosas, p. 185 y p. 166.

49) Torquemada, Jardín de Flores Curiosas, p. 134.

${ }^{50}$ Ver Zapata, Miscelánea, Tomo XI del Memorial Histórico Español, p. 32.

${ }^{51}$ Ver Zapata, Miscelánea, Tomo XI del Memorial Histórico Español, p. 255; por el contrario Zapata, Miscelánea, Tomo XI del Memorial Histórico Español, p. 247.

52 Mexía, Silva de varia lección, t. 1, p. 174.

${ }^{53}$ Ver Mexía, Silva de varia lección, t. 1, p. 377. 
ba de monstruosidades, «siendo tan graves y de tan grande autoridad, que nos obligan a creer que los hay", de donde él extrae la conclusión aparentemente constructivista: "[P] ero en fin se entiende que es verdad lo que está escrito» ${ }^{54}$.

¿Cuántos autores se tienen que presentar como autoridades para justificar la credibilidad de una cosa? En Torquemada, como se demuestra anteriormente, se confirma esta credibilidad: «con la autoridad de autores tan graves que la afirman por verdadera $\rangle^{55}$ o cuando "muchos autores dignos de fe escriben esto" ${ }^{56}$. Pero también a veces basta con una única autoridad especialmente destacada como Aristóteles ${ }^{57}$. También la de Hipócrates es suficiente para infundir de credibilidad las "cosas maravillosas» ${ }^{58}$. Para que "una cosa que tan fuera va de todos los límites de la razón y de naturaleza» ${ }^{59}$, se pueda tener por verdadera, hay que presentar a determinados autores.Y lo que no ha dicho ningún autor que se pueda considerar serio, debe ser rechazado por ser "ficción del vulgo" ${ }^{60}$. Mexía acentúa "que ninguna cosa digo ni escrivo que no la aya leído en libro de grande auctoridad, como las más vezes alegaré» ${ }^{61}$. Por tanto, quien quiera desestimar lo que lee, debe tener en cuenta ante todo la mencionada autoridad, pues no todo lo que una única persona no entiende, puede ser automáticamente considerado falso. Precisamente cuando algo es enunciado pareciendo realmente inverosímil, la mención de autores fidedignos es tanto más necesaria. La frecuencia de dichas menciones, debe aumentar, según Mexía, en la misma proporción que el grado de inverosimilitud $^{62}$, ya que en principio no se puede mencionar ni lo más interesante si no está corroborado por el informe de una autoridad ${ }^{63}$. Parece evidente que las Crónicas españolas disfrutan especialmente de credibilidad $^{64}$ y lo mismo sucede con los historiadores; que por ejem-

\footnotetext{
${ }^{54}$ Torquemada, Jardín de Flores Curiosas, p. 126.

55 Torquemada, Jardin de Flores Curiosas, p. 117.

${ }^{56}$ Torquemada, Jardín de Flores Curiosas, p. 113.

${ }^{57}$ Ver Torquemada, Jardín de Flores Curiosas, p. 117.

58 Torquemada, Jardín de Flores Curiosas, p. 189.

59 Torquemada, Jardín de Flores Curiosas, p. 156.

6i) Torquemada, Jardín de Flores Curiosas, p. 413.

${ }^{61}$ Mexía, Silva de varia lección, t. 1, pp. 164-165 y p. 177.

${ }^{62}$ Mexía, Silva de varia lección, t. 1, p. 721.

${ }^{63}$ Mexía, Silva de varia lección, t. 1, p. 709.

${ }^{64}$ Torquemada, Jardín de Flores Curiosas, p. 118.
} 
plo, no haya habido ningún historiador que al término de la Guerra de Troya haya informado sobre las amazonas es, según la opinión de Torquemada, razón para dudar de su subsistencia ${ }^{65}$.

¿Se puede, por tanto, deducir de ello que básicamente los historiadores de la Antigüedad o autores como Aristóteles y Hipócrates gozan de la mayor autoridad? ¿A quiénes se les debe conceder más autoridad: a los autores contemporáneos o a los autores de la Antigüedad? Rallo Gruss diferencia entre Mexía, el cual reúne de la Antigüedad lo que puede ser de interés para el lector contemporáneo ${ }^{66}$; Torquemada, que pone de relieve precisamente todo eso, de lo que todavía en la Antigüedad no se ocupaban, y Zapata, al que básicamente le interesa el propio presente ${ }^{67}$. Por eso, Zapata desprecia a los autores de la Antigüedad por "mentirosos antiguos ${ }^{68}$ y se enfada con aquellos que consideran su propia época menos intelectual y creadora que las épocas pasadas. Se lo argumenta de la siguiente manera: «En las ciencias y artes hace el tiempo de agora al antiguo grandísima ventaja ${ }^{69}$. Así puede presentar, con plena confianza en lo que dice, que su propia opinión es superior a la de la Antigüedad: «Desto dice Plutarco [...]; mas digo yo que $[\ldots]\rangle^{70}$. Torquemada decide del mismo modo, cuando menciona el ejemplo del autor de la Antigüedad Ptolomeo, el cual como geógrafo, tuvo que registrar las tierras como "no conocidas ni descubiertas", que conoce la época moderna por experiencia propia debido a "que han visto, andado y caminado y navegado tanto" ${ }^{71}$. Que Torquemada no decide así por estar en un apuro, sino que conoce el latín y la cultura de la Antigüedad, lo demuestran sus alusiones a las obras de esta época y sus citas literales repartidas en toda su obra ${ }^{72}$.

Si los autores garantizan más o menos la verdad según su autoridad, ¿cómo se decide entonces cuando un autor cristiano se opone a otro autor pagano? López Poza menciona una doctrina muy extendi-

${ }^{65}$ Torquemada, Jardín de Flores Curiosas, p. 135.

${ }^{66}$ Lerner, 1993, ha demostrado, con cuánta frecuencia Mexía echa mano además de las fuentes españolas.

${ }^{67}$ Ver Rallo Gruss, 1984, p. 180.

68 Zapata, Miscelánea, Tomo XI del Memorial Histórico Español, p. 21.

69 Zapata, Miscelánea, Tomo XI del Memorial Histórico Español, pp. 350-351.

70 Zapata, Miscelánea, Tomo XI del Memorial Histórico Español, pp. 1-2.

${ }^{71}$ Torquemada, Jardín de Flores Curiosas, p. 390.

${ }^{72}$ Ver Rodríguez Cacho, 1988, p. 68. 
da tras el Concilio de Trento por los representantes de la ortodoxia católica, sobre todo por Karl Borromäus y los jesuitas, según la cual, recurrir a autores paganos de la Antigüedad está de más, ya que los Santos Padres son lo suficientemente dignos de confianza en cuanto a la doctrina y la elegancia estilística ${ }^{73}$. Ante el trasfondo de semejante doctrina y del peligro por parte de la Inquisición, Torquemada da el siguiente consejo que se contradice con su práctica: «D] ejemos los autores y filósofos gentiles y sigamos solamente a los cristianos» ${ }^{74}$. En lo que a los sátiros se refiere, prefiere el testimonio del Santo Padre Hieronymus al de Pomponius Mela ${ }^{75}$. Para desvanecer la última duda, le parece indispensable que uno sea teólogo y experto en la interpretación de la Biblia ${ }^{76}$. Puesto que la Biblia es la máxima autoridad, no se fía del criterio de los paganos infieles «de las cosas divinas y principalmente de Dios y de su omnipotencia [... de su divina providencia universal en todas las cosas ${ }^{77}$. Del mismo modo superpone Mexía la autoridad de la Biblia sobre la de cualquier autor no cristiano $^{78}$. De todos modos, los autores no cristianos le parecen creíbles, allí donde faltan testimonios de autores cristianos ${ }^{79}$. Pérez de Moya va un poco más allá al observar que la autoridad religiosa no siempre es segura, pues ocurre a menudo que lo no religioso se presenta disfrazado de religiosidad; por eso en su escrito persigue el propósito «de no ser engañado por aquellos que disfrazándolo de religión recomiendan tonterías y que con la excusa de la santidad montan engaños dignos del demonio» ${ }^{80}$. Este cuidado es comprensible teniendo en cuenta la cantidad de corrientes heterodoxas contemporáneas y es por eso, que en principio, ya no se puede contar con mayor fiabilidad y autoridad por parte de los autores religiosos. La verdad absoluta se la reserva a Dios, de ahí que Torquemada, resignado, enuncie: «Dejemos estos secretos de naturaleza para sólo aquel que los hace» ${ }^{81}$. Uno se

${ }^{73}$ Ver López Poza, 1990, p. 63.

${ }^{74}$ Torquemada, Jardín de Flores Curiosas, p. 104 y pp. 252-253.

${ }^{75}$ Torquemada, Jardin de Flores Curiosas, p. 128.

${ }^{76}$ Torquemada, Jardin de Flores Curiosas, p. 208.

${ }_{77}$ Torquemada, Jardín de Flores Curiosas, p. 355.

${ }^{78}$ Mexía, Silva de varia lección, t. 1, p. 613.

${ }^{79}$ Mexía, Silva de varia lección, t. 1, p. 769.

${ }^{80}$ Pérez de Moya, Silva Eutrapelias. Traducción de la primera edicción de Valladolid 1557, p. 43.

${ }^{81}$ Torquemada, Jardín de Flores Curiosas, p. 202 y p. 453. 
tiene que dar por satisfecho con fragmentos de la verdad y se tiene que tener en cuenta que gran parte de ella permanece oculta ${ }^{82}$. Torquemada interrumpe continuamente sus indagaciones puesto que no verterán más luz sobre la verdad ${ }^{83}$.

En Mexía se da muchas veces el caso en el que predomina la inseguridad ante la diversidad de opiniones y la falta de una autoridad decisiva: «En esta confusión y variedad de opiniones, yo no sé quál me tuviesse por más verdadera, ni en la verdad se puede dar regla ni término cierto ${ }^{84}$. Además al encontrarse ante las opiniones opuestas de dos autoridades reconocidas por igual, es incapaz de hallar la verdad ${ }^{85}$. Aun cuando presenta las diferentes opiniones de los astrólogos referente a la edad del mundo, prescinde de reclamar la verdad y se ve obligado, en vista de su incompetencia, a ceder al lector la tarea de buscar la verdad. "Cada uno le dé el crédito y auctoridad que quisiere, que, cierto, no la dexa de tener. $»^{86}$ En Torquemada es, finalmente, más que una captatio benevolentiae, cuando permite decir a un conversante: "Cierto hay tantas razones para poder seguir cualquiera de las opiniones dichas, que lo mejor será no confundir el entendimiento ${ }^{87}$. En otra cita ${ }^{88}$ hace al mismo hablante retractarse en interés de la objetividad con la opinión «de varones tan sabios y tan excelentes como hay de la una parte a de la otra». «Solamente os he referido lo que hay por parte de los unos y de los otros.» Puesto que, aparentemente, todo lo demás no tiene nada que ver con el esclarecimiento objetivo de la verdad, sino que depende de la inclinación personal, sugiere: "Vosotros podréis inclinaros a la parte que mejor os pareciere», para finalmente acabar por expresar su opinión subjetiva: «A mi entendimiento, hay tantas razones por una parte y por otra, que casi me parece una cuestión indeterminable».

${ }^{82}$ Torquemada, Jardín de Flores Curiosas, p. 272.

${ }^{83}$ Torquemada, Jardín de Flores Curiosas, p. 140.

${ }^{84}$ Mexía, Silva de varia lección, t. 1, p. 528.

${ }^{85}$ «Lo qual visto por mí, he tomado por más sano consejo no querer yo apurar esto, pues no basta para ello, sino poner ambas cuentas y de los más principales auctores" (Mexía, Silva de varia lección, t. 1, p. 385).

${ }^{86}$ Mexía, Silva de varia lección, t. 1, p. 523; ver también «En esto la experiencia nos podría sacar de dubda, si alguno muy curioso lo quisiesse hazer; en tanto, tendrá cada uno la opinión que quisiere, pues va en ello muy poco» (Mexía, Silva de varia lección, t. 1, p. 383).

${ }^{87}$ Torquemada, Jardín de Flores Curiosas, p. 221.

${ }^{88}$ Torquemada, Jardín de Flores Curiosas, p. 375. 
Resumiendo podemos manifestar que los autores de las citadas colecciones no solamente tenían que presentar como nuevas las materias de las autoridades de la Antigüedad, sino que su problema era precisamente encontrar autoridades que confirmaran la verdad de lo que ellos contaban. La detallada controversia acerca de la garantía de la verdad de las diferentes fuentes no puede despistar de cuán dudosas les parecían las diferentes fuentes. La vía de argumentación escepticista que se toma con ello, nos recuerda a las dudas de Descartes, el cual, igualmente aceptaba metódicamente, que podría ser confundido por diferentes partes. Mientras que a Descartes le interesaba la capacidad de verdad de la Metafísica, es decir, la disciplina menos comprobable de la Filosofia, son, también para nuestros autores, los fenómenos menos comprobables y más extraordinarios, para los que se busca una garantía de verdad; ya que son de interés por cuanto se presentan como realidad y no como ficción, de acuerdo al convenio del género. Como Descartes, nuestros autores deben justificar que saben diferenciar la verdad de las historias inventadas y de las mentiras.

Se ha mostrado que una sola prueba como justificante de la verdad no parece suficiente, sino que al contrario, cuantos más testimonios se tengan, más ventajoso será. De todas formas, la experiencia propia debe ser completada o incluso sustituida por la ajena, cuando choca con barreras locales y temporales. Por eso, a la verdad se llega no sólo a través de dicha experiencia propia, sino también a través de la ajena, que se puede extraer de los libros o de lo que la gente dice. Lo último, la mayoría de las veces, no sólo ya no resulta extraordinario, sino que a la vez, poco seguro; por ello, no se debe creer en las habladurías de la gente. Tampoco disponen de mayor credibilidad los que ocupan una posición social más alta, incluso a veces, se debe dudar de lo que dicen informantes normalmente fidedignos. Cuando un hecho se confirma por el mayor número posible de autores serios, entonces uno lo tiene que considerar como verdadero; y cuanto más extraordinario sea su contenido, mayor tendrá que ser la cantidad de autores que lo confirmen.

¿A qué autores se les toma en serio? A Aristóteles, Hipócrates y a los historiadores de la Antigüedad, a quienes en algunas citas se prefiere, en otras se les rechaza por «mentirosos antiguos». Al juicio de Plutarco se le contrapone el propio, mientras que Ptolomeo es descalificado ya sólo a causa de la cantidad de tierras nuevas descubiertas. A esto se añade, que los autores cristianos son superiores a los paga- 
nos y lo que dice un Hieronymus, tiene más peso que lo que dice un autor de la Antigüedad. Pero debido a que la autoridad de textos cristianos se limita al campo religioso, es decir, que la autoridad ya no es absoluta $y$, por añadidura, un autor aparentemente religioso puede resultar un impostor, se pide también precaución frente a la autoridad religiosa.

Uno tiene la impresión de que se destruye cada autoridad tradicionalmente segura. Es especialmente interesante que ocurra esto en un discurso en donde se intenta precisamente lo contrario. Al seguir leyendo, uno se da cuenta de que en la medida en la que se proponen fuentes fidedignas, se les va desacreditando su autoridad; esto se hace especialmente visible cuando en un texto aparece la inseguridad, debido a dos opiniones opuestas o cuando se le encomienda al lector la tarea de encontrar por sí mismo la verdad. Como última autoridad queda la del sujeto correspondiente que, por ejemplo, prescinde de tomar una decisión o se puede dejar guiar por su inclinación personal. La última ancla segura, de la que se puede partir para buscar la verdad, es el sujeto, el cual puede decidir qué construcción de verdad prefiere. 


\section{BibliografíA}

\section{Literatura Básica}

Mexía, P., Silva de varia lección, ed. A. Castro, Madrid, Cátedra, t. 1, 1989; t. 2 , 1990.

Pérez de Moya, J., Silva Eutrapelias. Traducción de la primera edición de Valladolid 1557, ed. C. Clavería, Prólogo de V. Infantes y C. Baranda, Barcelona, Delstre's, 1996.

Torquemada, A. de, Jardín de Flores Curiosas, ed. G. Allegra, Madrid, Castalia, 1982.

Zapata, L., Miscelánea, Tomo XI del Memorial Histórico Español, Real Academia de la Historia, Madrid, Imprenta Nacional, 1859.

\section{Literatura Secundaria}

Castro, A., Introducción a Silva de varia lección, Madrid, Cátedra, 1989-1990. INFANTES, V., "De 'officinas' y 'polyantheas': los diccionarios secretos del siglo de oro", en Homenaje a Eugenio Asensio, Madrid, Gredos, 1988, pp. 243-257.

- Prólogo en Juan Pérez de Moya, Silva Eutrapelias, Barcelona, Delstre's, 1996.

Johnston, M. D., «La retórica del saber en el Jardín de flores curiosas de Antonio de Torquemada", Journal of Hispanic Studies, 3, 1978, pp. 69-83.

Jolles, A., Einfache Formen, Darmstadt, Wissenschaftliche Buchgesellschaft, (2. ed.), 1958.

Lausberg, H., Handbuch der literarischen Rhetorik, München, Hueber, (2. ed.), 1973.

Lerner, I., "Autores y citas españolas en la Silva de Mexía», Filología, 26, 1993, pp. 109-120.

López PozA, S., «Florilegios, polyantheas, repertorios de sentencias y lugares comunes. Aproximación bibliográfica», Criticón, 49, 1990, pp. 61-76.

Prieto, A., La prosa española del siglo XVI, t. 1, Madrid, Cátedra, 1986.

Rallo Gruss, A., "Las misceláneas: conformación y desarrollo de un género renacentista", Edad de Oro, 3, 1984, pp. 159-180.

- La prosa didáctica en el siglo XVI, Madrid, Taurus, 1987.

Rodríguez Cacho, L., "La frustración del humanista escribiente en el siglo XVI: el caso de Antonio de Torquemada", Criticón, 44, 1988, pp. 61-73.

- «La selección de lo curioso en 'silvas' y 'jardines': notas para la trayectoria del género», Criticón, 58, 1993, pp. 155-168. 